\title{
Acute Comparative Effect of Foam Rolling and Static Stretching on Range of Motion in Rowers
}

\author{
Alfonso Penichet-Tomas (D), Basilio Pueo*(D), Marta Abad-Lopez and Jose M. Jimenez-Olmedo (D) \\ Physical Education and Sports, University of Alicante, 06390 Alicante, Spain; alfonso.penichet@ua.es (A.P.-T.); \\ mal50@gcloud.ua.es (M.A.-L.); j.olmedo@ua.es (J.M.J.-O.) \\ * Correspondence: basilio@ua.es
}

\begin{abstract}
Rowers' anthropometric characteristics and flexibility are fundamental to increase stroke amplitude and optimize power transfer. The aim of the present study was to analyze the effect of foam rolling and static stretching on the range of motion over time. Eight university rowers ( $24.8 \pm 3.4$ yrs., height $182.3 \pm 6.5 \mathrm{~cm}$, body mass $79.3 \pm 4.6 \mathrm{~kg}$ ) participated in an alternating treatment design study with two-way repeated measures ANOVA. The sit and reach test was used to measure the range of motion. Both in the foam rolling and in the static stretching method, a pre-test (T0), a post-test (T1), and a post-15-min test (T2) were performed. A significant effect was observed on the range of motion over time $(p<0.001)$, but not for time $\mathrm{x}$ method interaction $(p=0.680)$. Significant differences were found between T0 and T1 with foam rolling and static stretching $(p<0.001, d=0.4) ; p<0.001$, $d=0.6)$. The differences between $\mathrm{T} 0$ and $\mathrm{T} 2$ were also significant with both methods $(p=0.001$, $d=0.4 ; p<0.001, d=0.4)$. However, no significant difference was observed between T1 and T2 $(p=1.000, d=0.1 ; p=0.089, d=0.2)$. Foam roller and static stretching seem to be effective methods to improve the range of motion but there seems to be no differences between them.
\end{abstract}

Citation: Penichet-Tomas, A.; Pueo, B.; Abad-Lopez, M.; Jimenez-Olmedo, J.M. Acute Comparative Effect of Foam Rolling and Static Stretching on Range of Motion in Rowers. Sustainability 2021, 13, 3631. https://doi.org/10.3390/su13073631

Academic Editor: Damián Iglesias Gallego

Received: 17 February 2021

Accepted: 22 March 2021

Published: 24 March 2021

Publisher's Note: MDPI stays neutral with regard to jurisdictional claims in published maps and institutional affiliations.

Copyright: (c) 2021 by the authors. Licensee MDPI, Basel, Switzerland. This article is an open access article distributed under the terms and conditions of the Creative Commons Attribution (CC BY) license (https:// creativecommons.org/licenses/by/ $4.0 /)$.

Keywords: traditional rowing; flexibility; sports; performance

\section{Introduction}

Flexibility is the range of motion of a joint or set of joints and is influenced by their component muscles, tendons, ligaments, and bones [1]. An improvement in flexibility can lead to an increase in muscle extensibility, together with an increase in joint range of motion [2]. Effective myofascial release, a limited and restricted fascia stretching technique, is necessary to achieve an increased range of motion [3]. The fascia is the connective tissue that surrounds muscles, nerves, and blood vessels, and connects the structures of the body [4]. Myofascial release not only appears to increase range of motion and improve muscle recovery, but it is also known to improve muscle performance both before and after exercise [5]. Thus, increasing the range of motion in athletes of different sports is very important for coaches and physical trainers, always keeping in mind the different characteristics of the treatments depending on the type of exercise or sport [6].

Different methods have been used and investigated to increase joint range, such as static stretching, a passive exercise in which a given posture is attained very slowly, producing the sensation of muscular elasticity without inducing pain [2]. However, foam rolling and manual massages seem to influence the manipulated muscle more directly. Moreover, it appears to increase motor recovery with similar effects during warm-up and post-exercise [7]. Foam rolling is a self-myofascial release method that consists of manual therapy where the subject applies force on a foam roller [5]. The correct procedure is to apply the foam roller from the origin to the insertion of the muscles to treat [8]. Several studies have compared foam rolling and static stretching, as well as other myofascial release methods [9], finding that foam roller yields a greater increase in range of motion than static stretching and improves flexibility and recovery by reducing muscle pain [5]. Furthermore, foam rolling seems to improve muscle soreness and muscle function loss [10]. 
Similarly, this technique seems to increase anti-inflammatory proteins, reduce proteins that promote inflammation, favoring muscle recovery and better performance [11], and increases blood lactate clearance, and leads to regenerate psychological characteristics during short-term compacted tournaments [12].

The studies that have analyzed the effect of foam rolling on sports performance seem to conclude that it yields small improvements in strength compared to static stretching [8], which even can negatively influence maximal muscle strength and explosive strength performance if it is done before exercise [13]. The use of foam rolling and the effect of self-myofascial release in the range of motion in the subsequent muscle activation is acutely enhanced without a deficit in muscle performance $[14,15]$ and it also influences the reduction of fatigue after exercise. Less fatigue can allow athletes to increase training time and volume [16]. Furthermore, foam rolling does not induce meaningful strength performance deficits and can be used to improve the extensibility of the muscles without harm performance [17]. Some studies have shown that it can be a useful tool for athletes of ballistics sports with stretch-shortening movement parameters [18] as in swimming, cycling or rowing [19].

In rowing, there is a strong relationship between strength development and greater performance [20]. This fact in combination with greater anthropometric characteristics of rowers [21] are factors to increase stroke length and power. A rowing stroke is divided into catch, drive, finish, and recovery [22]. For adequate length at the catch, rowers have to reach out using arm length, body swing, and slide compression [23]. A greater range of motion in rowing allows the flexion of the trunk during the stroke to achieve the correct degree at the catch and also a greater stroke when the athlete enters the oar into the water [24]. The back muscles are relaxed to allow trunk flexion and the arms are stretched to achieve greater stroke amplitude. The psoas and iliacus flex the pelvis and hips, aided by the rectus femoris, to obtain the correct degree of trunk inclination [25]. The femoral biceps and the gastrocnemius contract while the knees are semi flexed. Ankle dorsiflexion is produced by the tibialis anterior.

Studies published to date have shown the influence of different myofascial release techniques on the range of motion around specific joints or simple movements. However, there is no scientific evidence of myofascial release on complex or multi-joint technical sports gestures such as rowing. Rowers capable of reaching further in the catch will produce longer strokes. It is therefore essential to determine which method yields the greatest and most enduring increase range of motion to obtain optimal length stroke. In this study, the static stretching and foam rolling methods were applied and assessed using the sit and reach test to measure the muscles involved in the rowing stroke since this test simulates the position of the rower in the catch. Thus, the aim of the present study was to determine which method yields the greatest improvement in range of motion over time.

\section{Materials and Methods}

\subsection{Participants}

Eight university male rowers participated in the study (age: $24.8 \pm 3.8$ years, height: $182.3 \pm 6.5 \mathrm{~cm}$, and body mass: $79.3 \pm 4.6 \mathrm{~kg}$ ). The rowers had $2-4$ years of experience and all were attending six $2 \mathrm{~h}$ training sessions per week at the time of the study. The study protocol conformed to the guidelines of ethical principles of the Declaration of Helsinki and was approved by the Human Research Ethics Committee of the University of Alicante (IRB UA-2020-07-21). Each subject signed a written informed consent before participation.

\subsection{Procedure}

An alternating treatment design was used to explore the alternation of two interventions with different protocols to improve the range of motion focused on the comparison of treatment condition outputs [26]. Data were collected on two different weeks, with a rest week in between, on the same day, and at the same time of day (TOD) at 20:00 $\mathrm{h}$ to minimize biological influence $[27,28]$. The same training load was also established in both 
intervention weeks without strenuous exercise and high-intensity physical activity was not performed the previous $24 \mathrm{~h}$. In the first week, participants performed one session of flexibility with foam rolling; in the second week, participants carried out static stretching. In both weeks, participants performed sit and reach tests before executing the exercises (pre-test, T0) to confirm the repeatability of the protocol across two separate weeks [29]. The sit and reach test was used to measure the range of motion immediately after executing the exercises (post-test, T1) and again after a 15 min recovery period (post 15-min test, T2). In each case, subjects performed three trials and the best score out of three was used for subsequent analysis. The sit and reach was used since this test simulates the position of the rower in the catch (Figure 1).
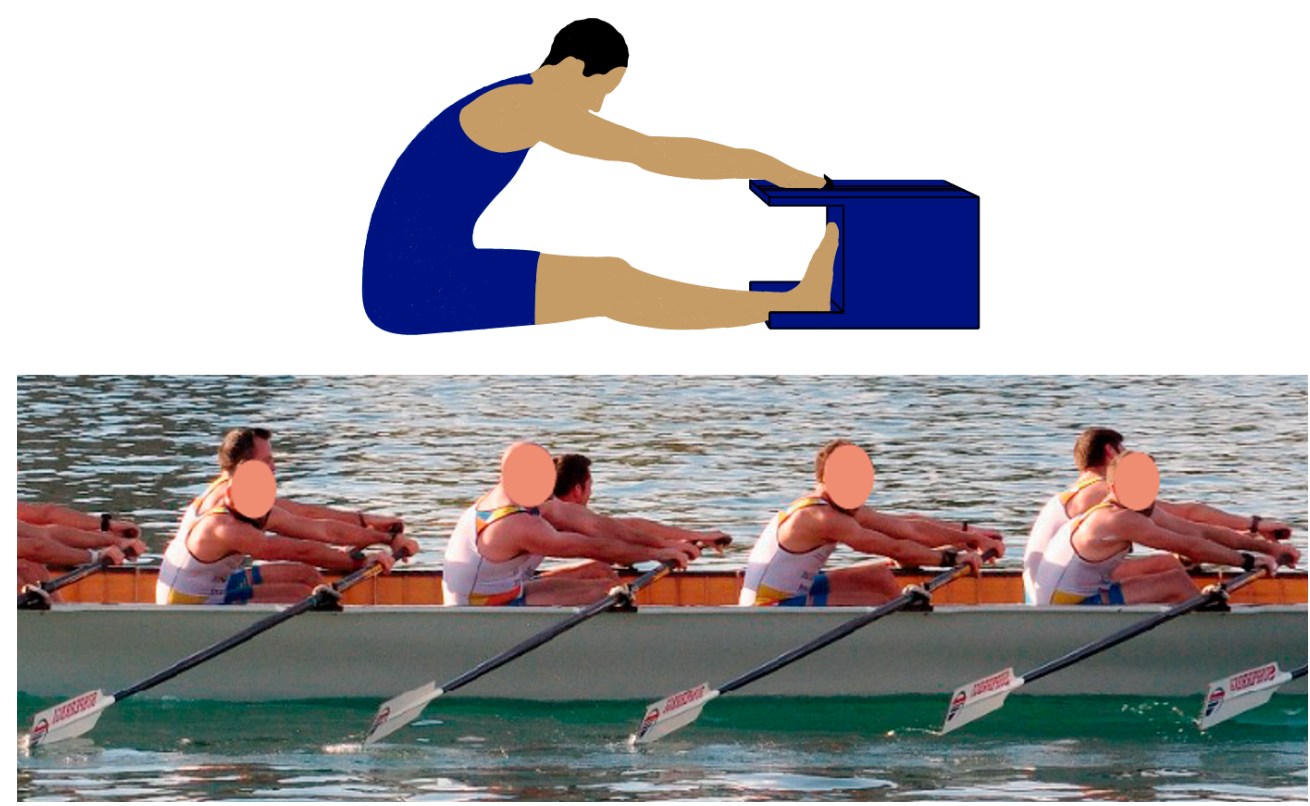

Figure 1. Body position in the sit and reach test and rowing body position in the catch.

The participants used a bumpy foam roller with a diameter of $12 \mathrm{~cm}$ and a $5 \mathrm{~mm}$ thick hollow plastic core covered with a $12 \mathrm{~mm}$ layer of dense foam. The protocol for foam rolling was 3 sets of $2 \mathrm{~s}$ repetition duration for each exercise, with a total duration of 30 s per set [17]. One repetition of foam rolling intervention consists of one distal rolling plus one subsequent proximal rolling movement [10]. The intensity with foam rolling was determined by the subjects' body weight as they used the foam roller, sliding it from the origin to the insertion of the muscle [14]. The muscles involved in the intervention were the gastrocnemius, hamstring, piriformis, lumbar and dorsal muscles.

The protocol for static stretching was 3 sets of $30 \mathrm{~s}$ for each exercise. Subjects were instructed to reach a position in which they noticed a slight but comfortable stretch with no pain. The muscles involved in the intervention were the same. The gastrocnemius stretched standing facing a wall taking a step forward with the right foot, with both palms on the wall, bending the right knee, leaning forward, and keeping the left leg straight and the left heel on the floor. To stretch the hamstring the rowers sat on the floor with right the leg extended in front and the left leg bent, to stretch forward with the right hand and touching their right toes. The piriformis muscle was stretched lying on the back with both feet flat on the floor, both knees bent, the ankle rest of the right leg over the knee of the left leg and pulling the left thigh toward the chest. The lumbar stretch was performed lying on their backs with their legs bent and knees pulled up to the chest. Finally, dorsal muscles were stretched standing straight with the feet hip-width apart, reaching the right arm over the head towards the left side while bending the side.

In both protocols each set lasted $5 \mathrm{~min}$ and $15 \mathrm{~s}$. There was a $5 \mathrm{~s}$ rest to change exercises and no rest between sets. The intervention in all muscles was unilateral except 
for the lumbar area. Rowers were supervised by the researchers to ensure the appropriate execution in both protocols.

The protocol of sit and reach was as follows: participants sat barefoot with their legs extended and the soles of their feet touching the box. If subjects bent their knees, the attempt was considered invalid. To eliminate differences due to each subject's anthropometry, the modified sit and reach test was used [30]. In the initial position, subjects sat with a straight back forming a 90 degrees angle to the hip. Their hands were placed one on top of the other on the box at the starting point (position 0). The distance measured was the maximum reached by the fingertips without separating the hands.

\subsection{Statistical Analysis}

Results were analyzed with Statistical Package for Social Sciences (SPSS v.26 for Windows, SPSS Inc., Chicago, IL, USA). Descriptive analysis was performed by the mean and standard deviation (SD) for all variables. The Shapiro-Wilk test was used to check sample normality, resulting in normal distribution. A two-way repeated measures ANOVA (T0, T1 and T2) was performed to compare differences between foam roller and static stretching methods. Any significant $F$ ratios were followed by Bonferroni's post-hoc analysis. Statistical significance was set at $p<0.05$. The Cohen's $d$ was used as a measure of the effect size of differences between T0, T1 and T2 and interpreted according to Cohen's thresholds: small $(d<0.3)$, medium $(d=0.3-0.4)$, large $(d=0.5-0.6)$, very large $(d=0.7-0.9)$ and extremely large $(d>0.9)$ [31].

\section{Results}

The improvement reached in the range of motion between $\mathrm{T} 0$ and $\mathrm{T} 1$ was $3.2 \pm 1.8 \mathrm{~cm}$ with the foam roller. This increase was maintained after $15 \mathrm{~min}$ since the difference between $\mathrm{T} 0$ and $\mathrm{T} 2$ was $3.3 \pm 1.9 \mathrm{~cm}$. The increase in range of motion between $\mathrm{T} 0$ and $\mathrm{T} 1$ was even greater with static stretching, $5.4 \pm 1.8 \mathrm{~cm}$. However, there was a decrease in range of motion in T2, $3.8 \pm 2.1 \mathrm{~cm}$ (Figure 2).

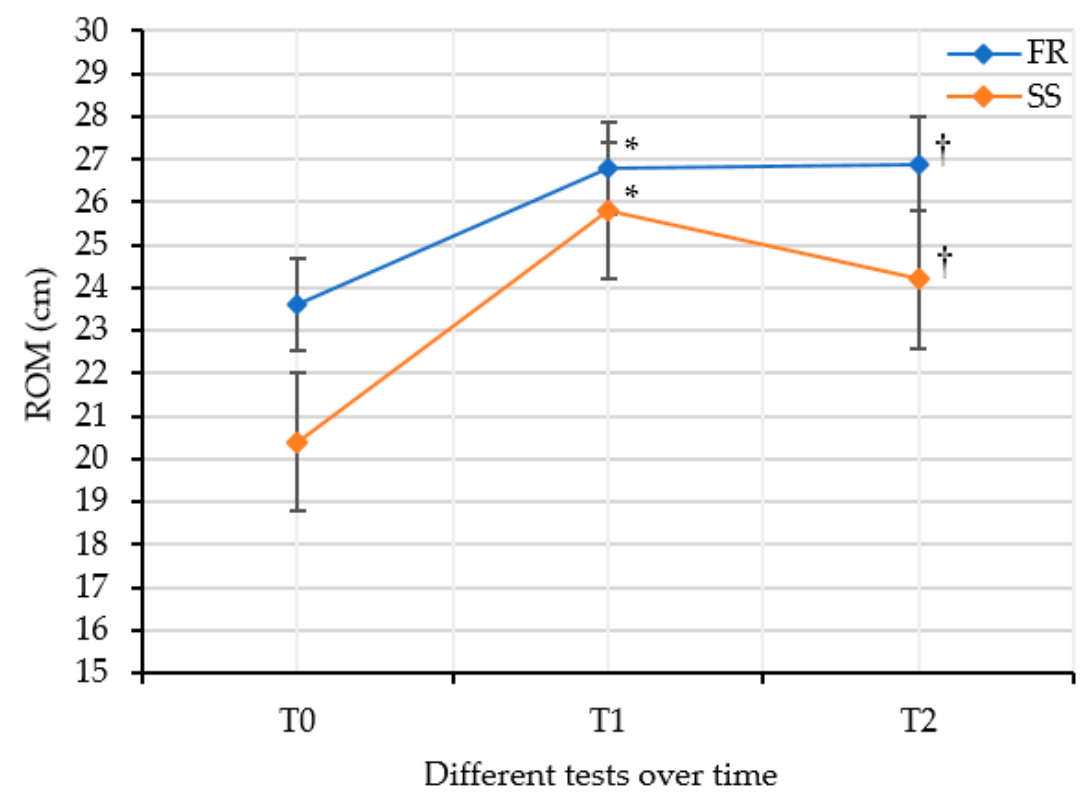

Figure 2. Comparative effect of FR (foam rolling) and SS (static stretching) in range of motion (cm) before intervention (T0), after intervention (T1) and after $15 \mathrm{~min}(\mathrm{~T} 2)$. * : significant differences $(p<0.05)$ between T0 and T1; + : significant differences $(p<0.05)$ between T0 and T2.

The two-way repeated measures analysis of variance revealed a significant main effect on the range of motion. There was a significant increased over the duration of the study $\left(\mathrm{F}(2,28)=47.451, p<0.001, \eta^{2}=0.772\right)$. However, there was no significant interaction of 
the protocols and time factors in the range of motion $\left(F(2,28)=2.965, p=0.680, \eta^{2}=0.175\right)$. The variations in the range of motion over time was not significantly different whether the foam roller was used or whether static stretching was performed. There was no significant main effect of protocol type on range of motion $\left(F(1,14)=0.323, p=0.579, \eta^{2}=0.023\right)$.

Post-hoc analysis revealed no significant difference in range of motion between protocols in T0 ( $p=0.455, d=0.4)$ (Table 1). Significant differences were found between T0 and T1 with foam roller and static stretching $(p<0.001, d=0.4) ; p<0.001, d=0.6)$ and a medium and large effect size was reported, respectively (Table 1). The differences between $\mathrm{T} 0$ and T2 were also significant with foam roller and static stretching $(p=0.001, d=0.4$; $p<0.001, d=0.4)$, although this time a medium effect size was reported in both protocols. However, no significant difference was observed between the T1 and T2 in any protocol. The range of motion was maintained with foam roller $(p=1.000, d=0.1)$ and the decrease was not statistically significant with static stretching $(p=0.089, d=0.2)$.

Table 1. Range of motion (ROM) performance across sessions and post hoc analysis of differences between tests.

\begin{tabular}{|c|c|c|c|c|c|c|c|c|c|}
\hline & \multicolumn{2}{|c|}{ T0 } & \multicolumn{2}{|c|}{ T1 } & \multicolumn{2}{|c|}{ T2 } & \multirow{2}{*}{$\begin{array}{c}\text { T0 vs. T1 } \\
p\end{array}$} & \multirow{2}{*}{$\begin{array}{c}\text { T1 vs. T2 } \\
p\end{array}$} & \multirow{2}{*}{$\begin{array}{c}\text { T0 vs. T2 } \\
p\end{array}$} \\
\hline & $\begin{array}{c}\text { ROM } \\
(\mathrm{cm})\end{array}$ & $95 \%$ CI & ROM (cm) & $95 \%$ CI & ROM (cm) & $95 \%$ CI & & & \\
\hline FR & $23.6 \pm 8.3$ & $16.7-30.6$ & $26.8 \pm 8.2^{*}$ & $19.9-33.6$ & $26.9 \pm 7.7^{\dagger}$ & $20.5-33.4$ & $<0.001$ & 1.000 & 0.001 \\
\hline SS & $20.4 \pm 8.6$ & $13.2-27.6$ & $25.8 \pm 8.4^{*}$ & $18.7-32.8$ & $24.2 \pm 8.9^{\dagger}$ & $16.7-31.7$ & $<0.001$ & 0.089 & $<0.001$ \\
\hline
\end{tabular}

T0: pre-test; T1: post-test; T2: post-15 min test; FR: foam rolling; SS: static stretching; ROM: expressed as mean \pm SD; $95 \%$ CI: confidence intervals; *: significant differences $(p<0.05)$ between T0 and T1; ${ }^{+}$: significant differences $(p<0.05)$ between T0 and T2.

\section{Discussion}

The aim of the present study was to determine which method yields the greatest improvement in range of motion over time in rowers because a wider range of motion allows the rower to perform a longer stroke in water [24]. The results of the present study indicate on a descriptive level that static stretching yielded a greater increase in range of motion immediately after the intervention $(p<0.001, d=0.6)$ and 15-min after $(p<0.001$, $d=0.4$ ). These results agree with previous research where static and dynamic stretching have also been effectively increased in flexibility [32,33]. The greatest increase with static stretching in our participants may be due to indirect involvement of the secondary musculature around the stretched muscles. In contrast, foam rolling exerts a more localized action, only involving a precise muscle. On the other hand, Su et al. [34] compared the effects of different stretching methods and observed statistically significant improvements in sit and reach with foam rolling compared to the other methods. This discrepancy may be due to the different types of exercises performed and the results measured, rendering a comparison of results difficult. However, static stretching before exercise can negatively influence athletic performance [13] while foam rolling does not appear to induce deficits in performance [17] and can be used to improve the extensibility of the muscles.

The use of foam rolling also induces significant increases in joint range of motion in the result of the present study (T0 vs. T1: $p<0.001, d=0.4$; T0 vs. T2: $p=0.001, d=0.4$ ) and the effect is comparable to stretching interventions [35]. Although the range of movement was lower in rowers in the present study, the effect has lasted longer in time, while with static stretching in the range decreased after the same time. However, this decrease was small, and no significant differences were found. Foam rolling also appears to be an effective strategy to obtain an acute improvement in flexibility and maintain the range of motion. It seems a useful tool for athletes seeking an acute increase in range of motion as part of a warm-up [36] and thus to prepare them for the subsequent rowing exercise. These improvements may be due to changes in pain modulation systems, minimizing the perception of pain and increasing tolerance to stretching [6]. However, it should be noted that the difference in the range of motion between methods may be due to the type of stretching employed, the duration of execution, the intensity applied in each method, or the 
muscle groups involved [8,9]. It is therefore advisable to control these aspects by designing protocols, since in short and explosive exercises the foam roller can negatively influence performance [37]. Thus, using a foam rolling during an active warm-up combined with dynamic stretching can reduce muscle stiffness and increase the range of motion [38].

The results of the present study should be interpreted with caution due to the small sample size and to the alternating treatment design of the study performed over two weeks by the same subjects. In addition, the pressure on the foam roller varied depending on the body weight and discomfort tolerance of each participant. Similarly, individual subjective sensations have not been objectively controlled in static stretching. Furthermore, control of the lumbar spine posture could offer very valuable additional information for the study. The greater the hip flexion, the greater the potential output of the larger muscle groups [39]. Nevertheless, when the lumbar spine is flexed, the load is greater on the disc than on the facet joints. It would be interesting to take this factor into account because in neutral posture the loads are distributed between facets and disks for optimal mechanics and load transfer [40].

Future research would be needed to explore the relationships between the variable of this study and postural control to reduce the risk of injury as much as possible. Due to the scientific scarcity and the controversy between the studies, future research should analyze the effects of long-term use and its benefits. It would also be interesting to see how a greater range of movement generated thanks to foam rolling can influence in a practical way. The existing literature seems to conclude that there will not be a negative effect on performance. Therefore, it would be interesting to analyze whether increasing the range of motion favors a greater stroke range in the water, and also produces greater power.

\section{Conclusions}

The study compares the acute effect of two different protocols on the range of motion in rowers by subjecting participants to an intervention with each method, one week apart. The results demonstrate that static stretching and foam rolling significantly increased range of motion, so both methods seem effective in increasing stroke range which is so important in rowing. However, no significant differences were found between both treatments in the acute effect on range of motion. Both methods can be considered to improve range of motion but treatments have to be carefully designed so as not to affect performance, as has been demonstrated in other studies with static stretching.

Author Contributions: Conceptualization, A.P.-T., B.P. and M.A.-L.; Data curation, M.A.-L.; Formal analysis, A.P.-T., B.P. and J.M.J.-O.; Funding acquisition, M.A.-L.; Investigation, A.P.-T., B.P. and J.M.J.O.; Supervision, J.M.J.-O.; Validation, J.M.J.-O.; Writing—original draft, A.P.-T.; Writing-review and editing, A.P.-T., B.P. and J.M.J.-O. All authors have read and agreed to the published version of the manuscript.

Funding: This research received no external funding.

Institutional Review Board Statement: The study was conducted according to the guidelines of the Declaration of Helsinki, and approved by the Institutional Review Board of University of Alicante (IRB No. UA-2020-07-21, date of approval: 8 August 2020).

Informed Consent Statement: Informed consent was obtained from all subjects involved in the study.

Data Availability Statement: The data presented in this study are available on reasonable request from the corresponding author.

Conflicts of Interest: The authors declare no conflict of interest.

\section{References}

1. Anderson, B.; Burke, E.R. Scientific, medical, and practical aspects of stretching. Clin. Sports Med. 1991, 10, 63-86. [CrossRef]

2. Chulvi-Medrano, I.; Masiá-Tortosa, L. La flexibilidad. Criterios básicos para su entrenamiento saludable aplicando la metodología pasivo-estática. J. Sport Health Res. 2012, 4, 11-22. 
3. Barnes, M.F. The basic science of myofascial release: Morphologic change in connective tissue. J. Bodyw. Mov. Ther. 1997, 1, 231-238. [CrossRef]

4. Sullivan, K.M.; Silvey, D.B.J.; Button, D.C.; Behem, D.G. Roller-massager application to the hamstrings increases sit-and-reach range of motion within five to ten seconds without performance impairments. Int. J. Sports Phys. Ther. 2013, 8, 228-236. [PubMed]

5. Beardsley, C.; Škarabot, J. Effects of self-myofascial release: A systematic review. J. Bodyw. Mov. Ther. 2015, 19, 747-758. [CrossRef] [PubMed]

6. Wiewelhove, T.; Döweling, A.; Schneider, C.; Hottenrott, L.; Meyer, T.; Kellmann, M.; Pfeiffer, M.; Ferrauti, A. A meta-analysis of the effects of foam rolling on performance and recovery. Front. Physiol. 2019, 10, 376. [CrossRef]

7. Kerautret, Y.; Di Rienzo, F.; Eyssautier, C.; Guillot, A. Selective effects of manual massage and foam rolling on perceived recovery and performance: Current knowledge and future directions toward robotic massages. Front. Physiol. 2020, 11, 1567. [CrossRef]

8. Halperin, I.; Aboodarda, S.J.; Button, D.C.; Andersen, L.L.; Behem, D.G. Roller massager improves range of motion of plantar flexor muscles without subsequent decreases in force parameters. Int. J. Sports Phys. Ther. 2014, 9, 92-102.

9. Škarabot, J.; Beardsley, C.; Štirn, I. Comparing the effects of self-myofascial release with static stretching on ankle range-of-motion in adolescent athletes. Int. J. Sports Phys. Ther. 2015, 10, 203-212.

10. Nakamura, M.; Konrad, A.; Yasaka, K.; Kiyono, R.; Onuma, R.; Yahata, K.; Sato, S. The acute effect of foam rolling on eccentricallyinduced muscle damage. Int. J. Environ. Res. Public Health 2021, 18, 75. [CrossRef]

11. Pablos, A.; Ceca, D.; Jorda, A.; Rivera, P.; Colmena, C.; Elvira, L.; Martínez-Arnau, F.M.; Valles, S.L. Protective effects of foam rolling against inflammation and notexin induced muscle damage in rats. Int. J. Med. Sci. 2020, 17, 71-81. [CrossRef] [PubMed]

12. Rahimi, A.; Amani-Shalamzari, S.; Clemente, F.M. The effects of foam roll on perceptual and performance recovery during a futsal tournament. Physiol. Behav. 2020, 223, 112981. [CrossRef]

13. Simic, L.; Sarabon, N.; Markovic, G. Does pre-exercise static stretching inhibit maximal muscular performance? A meta- analytical review. Scand. J. Med. Sci. Sports 2013, 23, 131-148. [CrossRef] [PubMed]

14. Macdonald, A.G.; Penney, M.; Mullaley, M.; Drake, C.; Behm, D.G.; Button, D.C. An acute bout of self myofascial release increases range of motion without a subsequent decrease in muscle activation or force. J. Strength Cond. Res. 2013, 27, 812-821. [CrossRef] [PubMed]

15. Kalichman, L.; Ben David, C. Effect of self-myofascial release on myofascial pain, muscle flexibility, and strength: A narrative review. J. Bodyw. Mov. Ther. 2017, 21, 446-451. [CrossRef] [PubMed]

16. Healey, K.C.; Hatfield, D.L.; Blanpied, P.; Dorfman, L.R.; Riebe, D. The effects of myofascial release with foam rolling on performance. J. Strength Cond. Res. 2014, 28, 61-68. [CrossRef] [PubMed]

17. Behm, D.G.; Alizadeh, S.; Hadjizadeh Anvar, S.; Mahmoud, M.M.I.; Ramsay, E.; Hanlon, C.; Cheatham, S. Foam rolling prescription: A clinical commentary. J. Strength Cond. Res. 2020, 34, 3301-3308. [CrossRef]

18. Morales-Artacho, A.J.; Lacourpaille, L.; Guilhem, G. Effects of warm-up on hamstring muscles stiffness: Cycling vs foam rolling. Scand. J. Med. Sci. Sports 2017, 27, 1959-1969. [CrossRef]

19. Held, S.; Siebert, T.; Donath, L. Changes in mechanical power output in rowing by varying stroke rate and gearing. Eur. J. Sport Sci. 2020, 20, 357-365. [CrossRef]

20. Gallagher, D.; Dipietro, L.; Visek, A.J.; Bancheri, J.M.; Miller, T.A. The effects of concurrent endurance and resistance training on 2,000-m rowing ergometer times in collegiate male rowers. J. Strength Cond. Res. 2010, 24, 1208-1214. [CrossRef]

21. Penichet-Tomás, A.; Pueo, B.; Jiménez-Olmedo, J. Physical performance indicators in traditional rowing championships. J. Sports Med. Phys. Fit. 2019, 59, 767-773. [CrossRef]

22. Smith, R.M.; Loschner, C. Biomechanics feedback for rowing. J. Sports Sci. 2002, 20, 783-791. [CrossRef] [PubMed]

23. Richardson, B. The catch. In Rowing Faster; Nolte, V., Ed.; Human Kinetic: Champaign, IL, USA, 2005.

24. Stutchfield, B.M.; Coleman, S. The relationships between hamstring flexibility, lumbar flexion, and low back pain in rowers. Eur. J. Sport Sci. 2006, 6, 255-260. [CrossRef]

25. Norris, C.M. Biomechanics of the lumbar spine. In Back Stability; Robertson, L.D., Mustain, E., Campbell, D., Feld, M., Schwarzentraub, M., Eds.; Human Kinetics: Champaign, IL, USA, 2000; pp. 14-42.

26. Portney, M.; Watkins, L. Foundations of Clinical Research: Applications to Practice; Pearson/Prentice Hall: Upper Saddle River, NJ, USA, 2002.

27. Ammar, A.; Chtourou, H.; Trabelsi, K.; Padulo, J.; Turki, M.; El, K.; Hoekelmann, A.; Hakim, A. Temporal specificity of training: Intra-day effects on biochemical responses and Olympic-Weightlifting performances. J. Sports Sci. 2014, 33, 358-368. [CrossRef] [PubMed]

28. Ammar, A.; Chtourou, H.; Souissi, N. Effect of time-of-day on biochemical markers in response to physical exercise. J. Strength Cond. Res. 2017, 31, 272-282. [CrossRef] [PubMed]

29. Doma, K.; Sinclair, W.H.; Hervert, S.R.; Leicht, A.S. Postactivation potentiation of dynamic conditioning contractions on rowing sprint performance. J. Sci. Med. Sport 2016, 19, 951-956. [CrossRef] [PubMed]

30. Hoffman, J. Flexibility. In Norms for Fitness, Performance and Health; Hoffman, J., Ed.; Human Kinetics: Champaign, IL, USA, 2006; pp. 97-105.

31. Cohen, J. Statistical Power Analysis for the Behavioral Sciences; Routledge: New York, NY, USA, 1988.

32. Curry, B.S.; Chengkalath, D.; Crouch, G.J.; Romance, M.; Manns, P.J. Acute effects of dynamic stretching, static stretching, and light aerobic activity on muscular performance in women. J. Strength Cond. Res. 2009, 23, 1811-1819. [CrossRef] 
33. McHugh, M.P.; Cosgrave, C.H. To stretch or not to stretch: The role of stretching in injury prevention and performance. Scand. J. Med. Sci. Sports 2010, 20, 169-181. [CrossRef]

34. Su, H.; Chang, N.J.; Wu, W.L.; Guo, L.Y.; Chu, I.H. Acute effects of foam rolling, static stretching, and dynamic stretching during warm-ups on muscular flexibility and strength in young adults. J. Sport Rehabil. 2017, 26, 469-477. [CrossRef]

35. Wilke, J.; Müller, A.L.; Giesche, F.; Power, G.; Ahmedi, H.; Behm, D.G. Acute effects of foam rolling on range of motion in healthy adults: A systematic review with multilevel meta-analysis. Sports Med. 2020, 50, 387-402. [CrossRef] [PubMed]

36. Skinner, B.; Moss, R.; Hammond, L. A systematic review and meta-analysis of the effects of foam rolling on range of motion, recovery and markers of athletic performance. J. Bodyw. Mov. Ther. 2020, 24, 105-122. [CrossRef] [PubMed]

37. Starzak, M.; Makaruk, H.; Makaruk, B.; Wiśniowski, P.; Annastarzak; Monikazajac. The foam rolling of quadriceps decrease the kinematics of step during a 30-m sprint run. J. Phys. Educ. Sport 2020, 20, 206-213. [CrossRef]

38. Hendricks, S.; Hill, H.; den Hollander, S.; Lombard, W.; Parker, R. Effects of foam rolling on performance and recovery: A systematic review of the literature to guide practitioners on the use of foam rolling. J. Bodyw. Mov. Ther. 2020, 24, 151-174. [CrossRef] [PubMed]

39. Francis, P. Loads on the bodies of rowers. In Rowing Faster; Nolte, V., Ed.; Human Kinetics: Champaign, IL, USA, 2011.

40. Bogduk, N. Clinical Anatomy of the Lumbar Spine ans Sacrum, 4th ed.; Elsevier: London, UK, 2005. 